\title{
4.6 Ästhetik des Schreckens: Der Schauerroman von Horace Walpole bis Ann Radcliffe
}

\author{
Michael C. Frank
}

\section{1. „Terrorist novel writing“: Die Anfänge des Schauerromans bei Horace Walpole}

In einem Handbuch zu Literatur \& Emotionen darf eine Gattung nicht fehlen, deren wesentliches Merkmal gerade darin besteht, dass sie mit literarischen Mitteln Emotionen erzeugt - zumindest, wenn wir der deutschen Gattungsbezeichnung ,Schauerroman‘ Glauben schenken. Der Begriff, Schauer' bezieht sich einerseits auf nasse Unwetter aller Art. Andererseits bezeichnet er jenes Frösteln, das außer von Regen-, Hagel- oder Schneeschauern auch durch das Gefühl wohligen Grusels hervorgerufen werden kann. Laut dem Grimmschen Wörterbuch manifestiert sich diese zweite Art des Schauers als „erschütterung des menschlichen körpers, schnell vorübergehende zitternde bewegung der haut, in folge von äuszern anlässen, besonders von kälte oder in folge von seelischen empfindungen“ (Grimm und Grimm 1999 [1893], 2324, im Original kursiv). Schauerromane, so die Implikation, erzeugen in ihren Leserinnen und Lesern eine vergleichbare Reaktion, wobei die Ursache für den Schauer hier freilich nicht von klimatischen Bedingungen abhängt, sondern allein in „seelischen empfindungen“ (ebd.) begründet liegt, die ihrerseits auf den Inhalt der betreffenden Texte zurückzuführen sind. Die Gattungsbezeichnung ,Schauerroman' sagt allerdings noch nichts darüber aus, wie genau dieser Effekt erzeugt wird. Was ist so schaurig an den Geschichten, die Schauerromane erzählen?

Auskunft darüber gibt ein Text aus der Zeit, in welcher der Schauerroman seine erste Blüte erlebte - und zwar in Großbritannien, wo die Gattung zwischenzeitig als ,Schreckensroman' bekannt war, bevor sich die heute geläufige Gattungsbezeichnung Gothic novel durchsetzte. In einem als Leserbrief formulierten Zeitungsessay beklagte sich ein anonymer Verfasser 1797 über die zeitgenössische Mode des „terrorist novel writing“ (Anonymus 2000 [1797]), die den literarischen Markt dominierte. Unmittelbarer Anlass für das satirische Beschwerdeschreiben waren die großen Erfolge Ann Radcliffes, deren mehrbändige Romanbestseller A Sicilian Romance (1790), The Romance of the Forest (1791), The Mysteries of Udolpho (1794) und The Italian (1797) zahlreiche Nachahmer auf den Plan gerufen hatten. Das Produkt dieser literarischen Mode beschreibt der anonyme Leser- 
briefschreiber wie folgt: „[...] novels, [...] in which it has been the fashion to make terror the order of the day, by confining the heroes and heroines in old gloomy castles; full of spectres, apparitions, ghosts, and dead men's bones.“ (Anonymus 1798 [1797], 223 [Hervorhebung im Original]) Wesentliche Bedeutung kommt demnach dem Schauplatz $\mathrm{zu}$ - dem finsteren mittelalterlichen Schloss, in dem sich die schauerlichen beziehungsweise schreckenerregenden Vorkommnisse ereignen. In dieser finsteren Umgebung trägt sich Übernatürliches zu. Mit gleich mehreren Wörtern wird das Motiv der Geistererscheinung beschrieben (,spectres, apparitions, ghosts“).

Es war der englische Parlamentarier Horace Walpole, der diesen gattungstypischen Schauplatz in die Romanliteratur einführte. Mit der Wahl eines mittelalterlichen, italienischen Settings begründete Walpole die traditionelle Vorliebe des frühen englischen Schauerromans für südeuropäische Handlungsorte und gotische Bauwerke, die Ann Radcliffe ebenso fortführte wie Matthew Gregory Lewis (in The Monk 1796), bevor der Schauerroman im Laufe des 19. Jahrhunderts verschiedene andere geografische Regionen für sich entdeckte (vgl. hierzu die vielen einschlägigen Beiträge in Byron und Townshend 2014). In der zweiten Auflage von 1765 gab Walpole seinem Roman den Untertitel A Gothic Story. Das englische Adjektiv Gothic war ursprünglich auf das Volk der Goten und dessen Sprache bezogen, seine Bedeutung wurde im 17. und 18. Jahrhundert aber zunehmend ausgedehnt (vgl. OED, „Gothic“). So bezeichnete es schließlich alles, was mit dem Mittelalter (dem ,finsteren Zeitalter`) verbunden war, insbesondere aber gotische Architektur. Es war diese Bedeutung, die Walpole vor Augen hatte, als er den Untertitel seines Romans formulierte. Obgleich sich die Verbindung des Adjektivs Gothic mit dem Genre des Schauerromans mithin bis 1765 zurückverfolgen lässt, gibt es nur eine Handvoll Autorinnen und Autoren, die die Walpolesche Gattungsbezeichnung für ihre Texte übernahmen (vgl. Clery 2002, 37, Anm. 2). Erst in den 20er, 30er Jahren des 20. Jahrhunderts sollte sich der Begriff Gothic novel einbürgern, nun allerdings als literaturwissenschaftliche Beschreibungskategorie (vgl. ebd., 37, Anm. 3).

Bis dahin galt terror als entscheidendes Distinktionsmerkmal der Gattung. Und wie der eingangs zitierte Leserbriefschreiber schon 1797 richtig erkannte, war für diesen Schrecken nicht allein der ,gotische“ Schauplatz verantwortlich, sondern auch und vor allem das, was sich dort ereignete. Der anonyme Verfasser unterschlug allerdings die wichtige Tatsache, dass in Bezug auf die schreckenerregenden Ereignisse im Schauerroman zwischen tatsächlichen und nur vermeintlichen Geistererscheinungen unterschieden werden muss. Bei Radcliffe beispielsweise erhalten scheinbar übernatürliche Geschehnisse spätestens am Romanende stets eine natürliche Erklärung, weshalb ihre Texte in der englischsprachigen Forschung der Kategorie des explained supernatural zugezählt werden 
(vgl. Clery 1995). Sir Walter Scott kritisierte dieses Erzählverfahren in einer Rezension des wenig bekannten Schauerromans Fatal Revenge; or, the Family of Montorio scharf: „[W]e disapprove of the mode introduced by Mrs. Radcliffe, and followed by [...] her [...] imitators, by winding up their story with a solution by which all the incidents, appearing to partake of the mystic and marvellous, are resolved by very simple and natural causes.“ (Scott 1810, 344) Im Gegensatz dazu bleibt das ,Wunderbare“ bei Horace Walpole bis zum Schluss als solches bestehen, weshalb Tzvetan Todorov - analog zum surnaturel expliqué - vom surnaturel accepté spricht (vgl. Todorov 1970, 47). Der Schauplatz ist hier in der Tat ein Spukschloss. Und Walpole bricht sogar noch eindeutiger mit dem mimetischen Prinzip der Wahrscheinlichkeit (vgl. Aristoteles 1997, Kap. 9, 29, 1451a-1451b): Nicht nur wird die Burg von Otranto von einem wahrhaftigen Geist heimgesucht, sondern dieser Geist hat noch dazu eine turmhohe Gestalt.

Wie sich im Verlauf der Erzählung erweist, handelt es sich bei dem riesenhaften Wiedergänger um Don Alfonso, den rechtmäßigen Fürsten von Otranto, der vor zwei Generationen während eines Kreuzzugs von seinem Kammerherrn Ricardo vergiftet wurde. Mit einem gefälschten Testament erschlich sich Ricardo daraufhin den Thron. Ricardos Enkel Manfred, der jetzige Fürst von Otranto, weiß von der Schuld seines Ahnen, ist jedoch nicht bereit, Gerechtigkeit walten zu lassen. Im Gegenteil hat er zusätzliche Schuld auf sich geladen. Als der legitime Thronfolger Frederic, der Marchese von Vicenza, bei einem Kreuzzug verwundet und gefangen genommen wurde, versuchte Manfred, die Situation für seinen eigenen Vorteil zu nutzen. Er bestach die Schutzbefohlenen von Frederics Tochter Isabella und ließ die junge Frau in seine Burg bringen, wo er sie mit seinem einzigen männlichen Nachkommen verheiraten will, um so die Erbfolge zu sichern. Die Romanhandlung setzt am Tag der Hochzeit mit einem ersten übernatürlichen Ereignis ein: Manfreds Sohn wird noch vor der Trauung von einem riesigen, aus dem Himmel fallenden Helm erschlagen.

Mit der Auferstehung Don Alfonsos in gigantischer Größe erfüllt sich ein Familienfluch. Einer „alten Prophezeiung“ zufolge wird der jetzige Fürst von Otranto die Herrschaft verlieren, sobald der wahre Eigentümer „zu groß geworden sein wird“, um die Burg zu bewohnen (Walpole 1998 [1764/1765], 17 [meine Übersetzung, M. C. F.]). Zu Beginn weiß nur Manfred, dass sein Großvater Ricardo den letzten rechtmäßigen Fürsten im Heiligen Land umgebracht hat. Auf der Heimreise in einen Sturm geraten, gelobte der Mörder und Usurpator damals, eine Kirche und zwei Klöster zu Ehren des Heiligen Nikolaus zu errichten, wenn er nur lebend ankomme - und wurde erhört. Seiner Familie sollte die Herrschaft über Otranto jedoch nur so lange gewährt werden, wie ihre männliche Linie fortbestehen würde. Und diese Zeit hat nun ihr Ende erreicht. Don Alfonso ist im wahrsten Sinne des Wortes „zu groß geworden“ (ebd. [meine Übersetzung, 
M.C.F.]). Im Anschluss an die Helm-Episode werden zunächst verschiedene Körperteile eines Riesen in Ritterrüstung gesichtet, dann lässt der Marchese von Vicenza ein Schwert von entsprechendem Ausmaß herbeitragen. Am Ende erscheint Alfonso in voller, monumentaler Gestalt inmitten der zu Ruinen zerfallenden Burg und identifiziert den wahren Erben. Die dergestalt inszenierte sprichwörtliche Größe Alfonsos hat zugleich eine metaphorische Bedeutung: Was hier zu groß geworden ist, ist die Last der Vergangenheit oder, genauer, die Bürde einer uneingestandenen Schuld. Alle Versuche Manfreds, den Untergang seines Geschlechts zu verhindern, scheitern. Nach dem Tod seines Sohnes will er Isabella zwingen, stattdessen ihn selbst zu heiraten. Manfred wird so zur ersten Verkörperung jener illegitimen (inzestuösen) Leidenschaft, welche in der weiteren Tradition des Schauerromans viele bunte Blüten getrieben hat. Die Flucht Isabellas durch die unterirdischen Passagen der Burg führt zugleich das Motiv der verfolgten Unschuld ein. Vor allem im Werk Radcliffes sollte die Figur der Jungfrau in Nöten zentrale Bedeutung erhalten. Bei Walpole liegt der Fokus der Erzählung stärker auf dem mächtigen Schurken selbst. Manfred wird immer mehr $\mathrm{zu}$ einem Gefangenen in seiner eigenen, von Don Alfonso heimgesuchten Burg. Hier bricht das Vergangene in die Gegenwart ein. Der Schrecken, der dadurch hervorgerufen wird, ist gleichbedeutend mit der Freudschen ,Wiederkehr des Verdrängten', die Verborgenes und Verheimlichtes auf un-heimliche Weise in Erscheinung treten lässt und das traute Heim auf diese Weise zu einem un-heimeligen Ort macht (vgl. Freud 1999 [1919], v. a. 232-237 und 254; für eine Anwendung der Freudschen Theorie auf The Castle of Otranto vgl. Tatar 1981, hier 171-174). So überrascht es wenig, dass das Konzept des Unheimlichen fester Bestandteil der literaturwissenschaftlichen Betrachtung des Schauerromans geworden ist (vgl. stellvertretend Jackson 1981, v. a. 63-71).

Es lohnt sich jedoch, nachträgliche Theoretisierungen vorübergehend auszublenden und zunächst einmal zu rekonstruieren, wie der Schrecken des Schauerromans in dessen eigener Zeit poetologisch reflektiert wurde. Und genau darum soll es in den folgenden Abschnitten gehen. Wie zu zeigen sein wird, ging dem Aufstieg des Schauerromans eine Neuentdeckung des Schreckens als affektive Quelle des ästhetischen Genusses voraus, für die vor allem Edmund Burkes Theorie des Erhabenen wegweisend war. Am Beispiel Horace Walpoles lässt sich diese poetologische Aufwertung - und Umdeutung - des Schreckaffekts gut nachvollziehen, da Walpole sie mit Verweis auf die klassizistische Tragödientheorie explizit thematisiert. $\mathrm{Zu}$ berücksichtigen sein wird ferner die politische Instrumentalisierung des Schreckens im Zuge der Grande Terreur. Denn in der jakobinischen Schreckensherrschaft glaubten mehrere frühe Kommentatoren eine direkte Ursache für den Boom des Schauerromans im ausgehenden 18. Jahrhundert zu erkennen - eine Verbindung, die schon die eingangs zitierte Rezension 
herstellt. Ihr Verweis auf Romane, die ,Schrecken auf die Tagesordnung setzen“ greift einen bekannten jakobinischen Slogan auf: Die Formel „Plaçons la terreur à l'ordre du jour“" wurde erstmals von dem ehemaligen Pfarrer Claude Royer am 30. August 1793 in einer Sitzung des Jakobinerclubs benutzt und später mehrfach aufgegriffen, am folgenreichsten von Bertrand Barère am 5. September 1793 gegenüber dem Komitee für Öffentliche Sicherheit (zit. nach Heuvel 1985, 108). Die Tatsache, dass die Kategorie der terreur um 1800 zwischen Poetik und Politik oszillierte, ist im vorliegenden Zusammenhang von einiger Bedeutung. Selbiges gilt für Radcliffes einflussreichen Versuch, zwischen terror und horror als zwei entgegengesetzten ästhetischen Prinzipien zu unterscheiden, mit dem ich diesen Beitrag abschließen möchte.

\section{2. „Terror, the author's principle engine“: Vom tragischen zum erhabenen Schrecken}

The Castle of Otranto erschien 1764 zunächst ohne Nennung von Walpoles Namen. In einem Vorwort wies der Autor den nachfolgenden Text als Übertragung eines italienischen Werkes aus, das vermutlich aus der Feder eines Priesters stamme. Das Original sei 1529 in Neapel gedruckt und nun in der Bibliothek einer alten katholischen Familie im Norden Englands aufgefunden worden. Möglicherweise stelle der Text ein Stück kontrareformatorischer Propaganda dar, das zeitgenössische Leser in ihrem Aberglauben bestärken sollte. Nach dieser Apologie des übernatürlichen Charakters der Romanhandlung sagt Walpole über seinen Text, er halte sich fast durchweg an die „Regeln des Dramas“ (Walpole 1998 [1764/1765], 6 [meine Übersetzung, M. C. F.]) - was die gesamte Herausgeberfiktion ad absurdum führt (denn warum sollte sich der besagte Priester am klassizistischen Regelkanon eines späteren Jahrhunderts orientiert haben?).

Mit Walpoles Verweis auf die dramatischen Regeln ist unter anderem die berühmte Passage aus der Poetik des Aristoteles gemeint, der zufolge die Tragödie im Zuschauer eleos und phobos hervorrufen soll, um mit den Mitteln des Theaters eine katharsis dieser Leidenschaften zu erreichen. Die Begriffe eleos und phobos wurden in der Aufklärung gemeinhin mit ,Mitleid' und ,Furcht' wiedergegeben, wohingegen Manfred Fuhrmann sie in seiner 1982 erschienenen Neuübertragung mit ,Jammer' und ,Schaudern“ übersetzt (Aristoteles 1997, Kap. 6, 19, 1449b). Laut Fuhrmann handelt es sich bei eleos und phobos um heftige Erregungszustände, die sich jeweils physisch manifestieren und die daher nicht als rein innere Empfindungen verstanden werden können. So habe phobos ursprünglich „ein durch Erschrecken bewirktes physisches Tun“ (Fuhrmann 1997, 162) bezeichnet, 
nämlich die Flucht vor einer Gefahrenquelle. Später habe sich die Bedeutung des Begriffes zwar auf den „Affekt des Erschreckens“ selbst verschoben, dieser Affekt lasse sich jedoch „nicht durch ,Furcht‘, eine lang anhaltende mildere Gestimmtheit, sondern eher durch ,Schrecken' oder ,Schaudern' angemessen wiedergeben“ (ebd., 163). Bei Walpole findet sich die aristotelische Paarung von eleos und phobos in folgender Formulierung wieder: „Never is the reader's attention relaxed. [...] Terror, the author's principal engine, prevents the story from ever languishing; and it is so often contrasted by pity, that the mind is kept up in a constant vicissitude of interesting passions." (Walpole 1998 [1764/1765], 6) Das Wort terror (Schrecken) kommt der Fuhrmannschen Deutung von phobos nahe. Im betreffenden Lemma des Oxford English Dictionary werden die zwei folgenden Hauptbedeutungen unterschieden: „1. The state of being terrified or greatly frightened; intense fear, fright, or dread. [...] 2. transf. a. The action or quality of causing dread; terrific quality, terribleness; spec. this action or quality in fiction, esp. in novel (or tale) of terror; also concr. a thing or person that excites terror; something terrifying.“ (OED, „Terror“) Dem deutschen Schrecken entsprechend kann terror demnach sowohl auf das Subjekt bezogen sein, das besagten Angstzustand empfindet (,erfüllte mich mit Schrecken'), als auch auf das Objekt, das diesen Zustand erzeugt (,der Schrecken des Todes'). Auffallend ist die starke Betonung des Schreckens in Walpoles Vorwort, die eindeutig auf Kosten des Mitleids geht. Während terror als Hauptmotor der Romanhandlung ausgewiesen wird, hat pity lediglich die Funktion, einen Kontrast zum Schrecken herzustellen, um so ein anregendes Wechselspiel der Leidenschaften zu erzeugen und die Aufmerksamkeit des Lesers wachzuhalten. Keine Rolle spielt in diesem Zusammenhang der aristotelische Gedanke einer kathartischen ,Reinigung، der besagten Affekte.

Die stärkere Gewichtung von phobos gegenüber eleos setzte bereits in der Tragödientheorie der französischen Aufklärung ein. Als entscheidenden Grund dafür identifiziert Helmut Keßler die verbreitete Auffassung, die Tragödie stehe im Dienste der moralischen Läuterung. Der Schrecken, so Keßler, wurde diesbezüglich als wirksamer erachtet als „das Mitleid mit dem Schicksal anderer, zumal nach Auffassung der Zeit die Angst als eines der bestimmenden Motive jedes menschlichen Handelns (bzw. Nicht-Handelns) galt“ (Keßler 1971, 157). In der Tat betont Louis de Jaucourt in seinem Encyclopédie-Eintrag zur terreur, der Zweck des aristotelischen phobos bestehe darin, den Zuschauer abzuschrecken: Das Negativbeispiel der tragischen Figur animiere uns dazu, unsere eigenen Leidenschaften zu zügeln, um einem ähnlichen Schicksal zu entgehen (Jaucourt 1765, 186). Dieselbe Interpretation des tragischen Schreckens findet sich in einer unvollendeten Abhandlung des Aphoristen Nicolas Chamfort (1741-1794), der zu Beginn seiner literarischen Karriere einige Erfolge als Theaterautor feierte. Chamforts posthum erschienene Ébauches d'une poétique dramatique enthalten einen 
Abschnitt zur terreur, dessen Anfangsabsätze weitgehend mit Jaucourts Encyclopédie-Artikel identisch sind, ihm aber die Unterscheidung zwischen terreur directe und terreur réfléchie hinzufügen (vgl. Chamfort 1824, 190-192). ,Unmittelbaren Schrecken' empfinden wir laut Chamfort dann, wenn wir Angst um eine Figur in einem Theaterstück haben. Er überkommt uns etwa in der Szene von Pierre Corneilles Rodogune (1645), in der Antiochus sich anschickt, aus einer vergifteten Schale zu trinken. In solchen Momenten ist unser Geist ganz vom Schicksal des tragischen Helden erfüllt: Wird er sterben oder wird er gerettet werden? Anders verhält es sich bei der terreur réfléchie. Einen solchen, reflexiven Schrecken' empfinden wir, sobald wir die fiktive Szene eines Theaterstückes auf unsere eigene reale Situation übertragen. In Voltaires Zaïre (1732) zum Beispiel stößt Orosman in einem Augenblick der Wut und der Eifersucht einen Dolch in das Herz seiner geliebten Zaire. Wissend, dass wir zu denselben Leidenschaften fähig sind, fürchten wir um uns selbst - und uns selbst. Und deshalb hält unsere affektive Reaktion auch deutlich länger an: Wir bleiben selbst nach Beendigung der Aufführung emotional erregt. Das aristotelische Konzept der katharsis impliziert in Chamforts Augen, dass phobos und eleos in der griechischen Antike als Schwächen betrachtet wurden, von denen man die Zuschauer heilen müsse. Dementsprechend hätten die klassischen Tragödien zumeist eine terreur directe angestrebt, die auf die Dauer des Stückes beschränkt bleiben sollte. Im Gegensatz dazu versuche das moderne Drama bewusst, Leidenschaften zu erregen, um sie für ,moralische Lektionen“ (leçons morales) zu nutzen: „Le théâtre modern ne prétend pas nous guérir de la pitié ni de la terreur, ni simplement se borner à exciter ces deux grandes affections en nous, pour le plaisir de nous faire verser des larmes et de nous épouvanter; mais il prétend s'en servir comme des deux plus puissans [sic!] ressorts pour nous porter à l'horreur du crime et à l'amour de la vertu.“ (ebd., 193) [„Das moderne Theater behauptet weder, uns von Mitleid oder Schrecken zu heilen, noch will es diese großen Leidenschaften nur in uns erregen, um uns das Vergnügen zu bereiten, Tränen zu vergießen und uns zu entsetzen; vielmehr gibt es vor, sie als Triebfedern zu nutzen, um uns zum Grausen vor dem Verbrechen und zur Liebe zur Tugend zu bringen.“ (M. C. F.)]

Grausen vor dem Verbrechen und Liebe zur Tugend - um diese ethischen Dispositionen im Publikum hervorzurufen beziehungsweise $\mathrm{zu}$ verfestigen, müssen sie affektiv vermittelt und gestützt werden. Und dafür nutzt die Tragödie vor allem den Schreckaffekt. Sehr deutlich wird die Umkehrung der aristotelischen Rangfolge von eleos und phobos in Baculard d'Arnauds Vorrede zu seiner 1770 entstandenen Tragödie Fayel. Während d'Arnaud den Mitleidsaffekt gänzlich vernachlässigt, apostrophiert er die Tragödie kurzerhand als „genre terrible“ (d'Arnaud 1795, vi [Hervorhebung im Original]). Seiner Ansicht nach sind seit der griechischen Antike nur noch die Engländer - zumindest in einigen Szenen - der 
eigentlichen Bestimmung der Tragödie nachgekommen, heftige Leidenschaften und insbesondere Schrecken $\mathrm{zu}$ erregen. Corneille, dem Gründungsvater des französischen Dramas, sei eine solche Wirkung hingegen nur noch an einer einzigen Stelle geglückt, nämlich im fünften Akt der Rodogune: „[C]'est-là [sic!] qu'il se rend maître de moi, me fait craindre, frissonner; je suis prêt à m'écrier; j'éprouve ce bouleversement des sens, tous ces divers orages qui doivent agiter Antiochus, Rodogune, Cléopatre, \&c.“ (ebd., vii) [,[H]ier gewinnt er Gewalt über mich, versetzt mich in Angst und Beben; ich bin kurz davor, aufzuschreien; ich empfinde dieselbe Überwältigung der Sinne, all diese Gewitter, die auch Antiochus, Rodogune, Kleopoatra etc. erschüttern.“ (M.C. F.)].

In seiner Betrachtung dieser Textstelle lässt Keßler unerwähnt, dass die hier beschriebene terreur, wenn überhaupt, nur noch partiell im Dienste der Tugenddidaxe steht (vgl. Keßler 1971, 157). Der mit dramatischen Mitteln hervorgerufene Schrecken erscheint weitgehend als ästhetischer Selbstzweck, eine lustvolle Empfindung, die ein Stück und seine Figuren überhaupt erst einprägsam macht. Von der aus Aristoteles' Poetik abgeleiteten terreur-Konzeption des Klassizismus ist nicht mehr viel übrig. Vielmehr befinden wir uns in einem anderen theoretischen Feld. Terreur steht hier mit Merkmalen in Verbindung, die d’Arnaud „Größe (grandeur)“ und „Erhabenheit (sublime)“ (d’Arnaud 1795, vii, Anm.) nennt. Das, was wir als terreur tragique bezeichnen könnten, ist mittlerweile von einer terreur sublime abgelöst worden, und genau an diesem Punkt setzt Walpole in seinem Vorwort zu The Castle of Otranto an. Der Schauerroman ist gewissermaßen ein neues genre terrible, das den Schrecken als Quelle des ästhetischen, erhabenen Genusses einsetzt.

\section{3. „The strongest emotion which the mind is capable of feeling ": Der erhabene Schrecken nach Edmund Burke}

Die Theorie des Erhabenen wird oft als Reaktion auf den klassizistischen Regelkonformismus verstanden. Wie das Beispiel d'Arnauds zeigt, gab es aber durchaus Überschneidungen. Beide Strömungen speisten sich zudem aus einer gemeinsamen Quelle, nämlich den 1674 veröffentlichten Euvres diverses von Nicolas Boileau. In ihnen enthalten ist neben Boileaus eigenem, eng an die antiken Regelpoetiken angelehntem Art poétique auch seine mit Einleitung und Anmerkungen versehene Übersetzung des pseudo-longinischen Traité sur le sublime ou du merveilleux dans le discours (vgl. Boileau-Despréaux 1674). Letzterer Text löste vor allem in England eine wahre Flut an Publikationen zum Erhabenen aus, während der das ursprünglich rhetorische Konzept in die Ästhetik überführt wurde (vgl. 
Monk 1960 [1935]). Bei Pseudo-Longin ist das Erhabene primär eine Eigenschaft menschlicher Rede. Das Konzept steht in seinem Text für das im metaphorischen Sinne ,Große‘ in Prosa und Poesie, in dem sich die Seelengröße des betreffenden Autors manifestiert. Der Begriff bezeichnet all das, was den Hörer nicht rational überzeugt, sondern affektiv berührt, ja: überwältigt (vgl. Longinus 1997, 5-7). Die dazu benötigten Grundfähigkeiten sind nach Pseudo-Longin naturgegeben; sie müssen jedoch durch eine entsprechende Kunstfertigkeit ergänzt werden, die rhetorisch geschult werden kann. Zu diesem Zweck erläutert der Autor am Beispiel antiker Klassiker verschiedene Strategien für eine erhabene Redeführung. Um zu belegen, dass dem Menschen die Bewunderung des Großen angeboren ist, verweist er dabei - wenn auch eher beiläufig - auf den erhabenen Charakter von Meeren und Vulkanausbrüchen (vgl. ebd., 89).

Im 18. Jahrhundert verschob sich der Akzent zunehmend von der bei PseudoLongin und Boileau betonten Großartigkeit und Suggestionskraft bestimmter Redner oder Dichter auf die erhabene Dimension natürlicher, politischer und kultureller Phänomene. Das Erhabene erschien nun als eine Eigenschaft, die gewissen Gegenständen ebenso inhärent ist wie deren Darstellungen in Literatur und Kunst. Dabei kristallisierte sich terror als Hauptauslöser erhabener Empfindungen heraus. Burkes Philosophical Enquiry into the Origin of Our Ideas of the Sublime and the Beautiful (1757, 2. Aufl. 1759) ist bei weitem nicht der einzige Traktat zum Thema, sollte sich aber als besonders wirkmächtig erweisen. Burke führt darin die - gegensätzlich verstandenen - Empfindungen des Erhabenen und des Schönen auf basale menschliche ,Leidenschaften“ (passions) zurück, die wir heute eher als ,Instinkte‘ bezeichnen würden (vgl. Burke 2004 [1757/1759], 85-88). Die sozialen Leidenschaften gewährleisten das Fortbestehen der menschlichen Spezies, indem sie uns die Gesellschaft anderer aufsuchen lassen. Sie äußern sich im Gefühl der Liebe (in ihren unterschiedlichen Erscheinungsformen und Intensitätsgraden) und wirken, indem sie Vergnügen bereiten. In entsprechender Weise wird das Überleben jedes Einzelnen durch Leidenschaften abgesichert, die im Dienste der Selbsterhaltung stehen. Sie wirken dadurch, dass sie in lebensbedrohlichen Situationen die Gefühle der Angst (fear) und des Schreckens (terror) in uns hervorrufen. Während nach Burke die sozialen Leidenschaften für die Empfindung des Schönen verantwortlich sind - paradigmatisch repräsentiert durch die von Männern empfundene Schönheit der Frau -, beruht das Erhabene auf den Vorstellungen von Schmerz und von Gefahr. Da der Selbsterhaltungstrieb die Grundbedingung dafür ist, dass die sozialen Instinkte überhaupt zur Wirkung kommen können (das Überleben des Einzelnen ist Voraussetzung für die Reproduktion der Spezies), geht Burke davon aus, dass dieser Trieb die stärksten Emotionen auslöst. Eine berühmte Passage zu den Quellen des Erhabenen lautet bei Burke: „Whatever is fitted in any sort to excite the ideas of pain, and danger, that 
is to say, whatever is in any sort terrible, or is conversant about terrible objects, or operates in a manner analogous to terror, is a source of the sublime; that is, it is productive of the strongest emotion which the mind is capable of feeling. [...] When danger or pain press too nearly, they are incapable of giving any delight, and are simply terrible; but at certain distances and with certain modifications, they may be, and they are delightful, as we every day experience.“ (ebd., 86)

Offenbar war Burke der Ansicht, dass Instinkte, die eine lebenswichtige Funktion haben, mit angenehmen Empfindungen verbunden sein müssen. Dabei lag es ihm freilich fern, eine masochistische Freude am Schmerz und an der Gefahr zu postulieren. Was uns ein intensives Vergnügen bereitet, ist Burke zufolge die Vorstellung von Schmerz und Gefahr, nicht eine Situation, in der wir tatsächlich von Schmerz und Gefahr betroffen sind (vgl. ebd., 97). Mit der Behauptung, Schmerz und Gefahr bereiteten - aus der Distanz betrachtet - ein noch intensiveres Vergnügen als das Schöne, bietet Burke eine Erklärung dafür an, warum sich der Mensch in Bild und Text so gerne mit dem Furchterregenden, Schrecklichen konfrontiert und warum sich nur ein Teil der Kunst- und Literaturproduktion auf eine anthropologische Freude am Schönen zurückführen lässt. Die sieben Jahre nach Erscheinen der Burkeschen Enquiry begründete Tradition der Gothic novel könnte als ein Beleg für diese These gedeutet werden. Fest steht, dass der Schauerroman in seinen Ursprüngen dasselbe zeitspezifische Vergnügen am ästhetisch erzeugten Schrecken zum Ausdruck bringt wie Burkes Essay, mit dem er überdies eng intertextuell verknüpft ist, wie noch zu zeigen sein wird.

\section{Terror zwischen Politik und Poetik}

Neben dem Diskurs des Erhabenen könnte es noch einen weiteren außerliterarischen Faktor gegeben haben, der zur Entstehung und zum Aufstieg des Schauerromans beitrug. Auf ihn deutet schon die Gattungsbezeichnung terrorist novel hin. Just in den 1790er Jahren, als in Großbritannien die terrorist novel boomte, erfuhr das Wort terror eine entscheidende semantische Erweiterung. Seit der Grande Terreur (1793-1794) war es eindeutig politisch konnotiert, was insbesondere auch für das von ihm abgeleitete Adjektiv terroriste gilt, das sich unmittelbar nach dem Sturz Robespierres von Frankreich aus verbreitete - als diskreditierende Bezeichnung für die Jakobiner (vgl. Heuvel 1985, hier 124; Walther 1990, hier 348). Im Jahr 1795 lässt sich das Substantiv terrorist erstmals im Englischen nachweisen. Die berühmte Stelle stammt aus einem Brief Burkes, der die jüngsten Ereignisse in Frankreich mit den Worten kommentierte: „Thousands of those Hell-hounds called Terrorists [...] are let loose on the people.“ (zit. nach $O E D$, „Terrorist“) 
Der politische Terrorismus schwingt in dem Titel Terrorist Novel Writing unweigerlich mit, auch wenn der Verfasser des Schreibens dies nicht weiter kommentiert. Ein weiterer Leserbrief aus demselben Jahr nutzt die Zweideutigkeit des Begriffes terrorist dagegen in ganz expliziter Weise. Das heute unter dem Titel The Terrorist System of Novel-Writing bekannte Schreiben an das Monthly Magazine betrachtet den Schauerroman als eine Nachahmung der Robespierreschen Terreur im Medium der (englischen) Literatur: „[S]o prone are we to imitation, that we have exactly and faithfully copied the system of terror, if not in our streets, and in our fields, at least in our circulating libraries, and in our closets.“ (Anonymus 2000 [1797], 299-300 [Hervorhebung im Original großgeschrieben]) Bei der Etablierung einer literarischen Schreckensherrschaft, so der Verfasser weiter, handele es sich um nichts Geringeres als eine literarische Revolution - eine Revolution allerdings, die nichts Neues hervorgebracht habe, sondern im Gegenteil eine kulturelle Regression in das Zeitalter der „Gespenster, Kobolde und Geister“ (ebd., 300 [meine Übersetzung, M. C. F.]) herbeigeführt habe. Infolge dieser Entwicklung sei es heute leichter denn je, als Schriftsteller zu Ruhm zu gelangen. Denn hierfür bedürfe es gar keiner literarischen Begabung. Die Leistung eines Autors werde allein danach bemessen, wie erfolgreich er seine Leserinnen und Leser in Schrecken versetze: „What has he [i. e. a writer] to do but build a castle in the air, and furnish it with dead bodies and departed spirits, and he obtains the character of a man of a most wonderful imagination, rich in imagery, and who has the wonderful talent of conducting his reader in a cold sweat through five or six volumes.“ (ebd., 300) Der Verfasser des Leserbriefs - der sein Schreiben ironisch mit ,A Jacobin Novelist' signiert - gibt vor, die Rückkehr in das Zeitalter der Schreckgespenster zu begrüßen, wofür er zwei Argumente vorbringt. Einerseits habe diese Entwicklung den literarischen Markt demokratisiert und für unbegabte Autoren geöffnet. Und andererseits habe sie zu einer dringend benötigten Wiederbelebung der Einbildungskraft geführt. Die zur Routine gewordene Beschreibung menschlicher Sitten habe sich erschöpft und der realistische Roman sein Motiv-Repertoire verbraucht. Folgerichtig verlangten die Leserinnen und Leser heute nach etwas, das nicht ihrem eigenen Alltag, sondern der Imagination eines Autors entsprungen sei. An dieser Stelle setzt der Verfasser die Engführung von politischer Schreckensherrschaft und literarischer Schreckenserzeugung fort: „[J]ust at the time when we were threatened with a stagnation of fancy, arose Maximilian [sic!] Robespierre, with his system of terror, and taught our novelists that fear is the only passion they ought to cultivate, that to frighten and instruct were one and the same thing, and that none of the productions of genius could be compared to the production of an ague." (ebd., 300)

Der Überzeugung, dass die Französische Revolution geradezu ursächlich für die Entstehung der Gothic novel gewesen sei, war auch der Marquis de Sade. In 
einer kurzen Passage seiner 1800 erschienenen Idée sur les romans spricht de Sade von einer neuen Gattung, die sich weitgehend auf die Merkmale Zauber (sortilège) und Trugbilder (fantasmagorie) reduzieren lasse, und er fügt hinzu, diese neue Gattung sei die notwendige Folge der revolutionären Erschütterungen, die ganz Europa erfasst hätten (vgl. Sade 1961 [1800], 31). Gerade in den fantastischen Elementen der neuen Gattung erkennt de Sade einen Effekt der Französischen Revolution. Angesichts des Leides, das reale Bösewichte (méchants) den Menschen zugefügt hätten, sei es schwer geworden, Romane zu schreiben, die mit den Schrecken der Wirklichkeit mithalten könnten; und so hätten die Romanciers die Hölle um Hilfe angerufen und sich ihre Stoffe im Land der Schimären besorgt (vgl. ebd., 31). Konkret nennt de Sade nur den Namen Radcliffe sowie den Titel Le Moine, wobei er abschließend anmerkt, dass er letzteren Roman den Werken Radcliffes in jeder Hinsicht vorzieht (vgl. ebd., 31).

In dem unter Zeitgenossen äußerst kontrovers diskutierten Monk des zum Zeitpunkt der Niederschrift erst neunzehnjährigen Matthew Lewis erkennen auch jüngere Analysen Referenzen auf das politische Geschehen in Frankreich. Dabei liegen solche Referenzen auf den ersten Blick fern. Thema des 1796 erschienenen Romans sind die sexuellen Verstrickungen und Verbrechen des allseits für seine Frömmigkeit und Keuschheit bewunderten Kapuzinermönchs Ambrosio. Von einer Agentin des Teufels verführt, verkommt Ambrosio zu einem gewissenlosen Vergewaltiger, Entführer und Mörder, der am Ende gar seine Seele an den Satan verkauft. Hauptschauplatz ist zwar ein Madrider Klosterkomplex zur Zeit der spanischen Inquisition, doch haben einige Szenen durchaus eine aktuelle Resonanz. An seinem Höhepunkt beschreibt der Roman, wie eine aufgebrachte Menschenmasse die Priorin des Nonnenklosters lyncht und schließlich - in einem kollektiven Blutrausch - in das Gebäude eindringt, um auch alle Nonnen zu töten und den Komplex in Brand zu stecken. Ausgangspunkt ist die berechtigte Empörung darüber, dass die schwangere Nonne Agnes im unterirdischen Gewölbe gefangen gehalten, gefoltert und vermeintlich getötet wurde. Doch selbst nachdem die sadistische Priorin sprichwörtlich zu Brei zertrampelt worden ist, eskaliert die Gewalt so lange, bis die Gemäuer des Klosters über der wütenden Meute zusammenstürzen, die Revolution also ihre eigenen Kinder verschlungen hat.

Unter anderem hierauf verweist Ronald Paulson, wenn er in einem klassisch gewordenen Aufsatz Gothic novels als metaphorische Repräsentationen der Ereignisse in Frankreich liest. Aus einer solchen Perspektive erscheint die MobEpisode aus The Monk als eine Allegorie auf die Erstürmung der Bastille (vgl. Paulson 1981, 534-535). Dieser Ansatz ist mit der Tatsache vereinbar, dass die zentralen Motive des Schauerromans bei Ausbruch der Revolution längst existierten, was zeitgenössische Kommentare übersehen, die die Gattung erst bei Radcliffe und Lewis beginnen lassen. Der Schauerroman stellte in seiner bereits 
bestehenden Form eine Schreibweise bereit, die für die metaphorische Reflexion politischer Gewalt aktualisiert und erweitert werden konnte und die nicht - wie von de Sade behauptet - neu erfunden werden musste. In diesem Sinne liest auch Patrick Brantlinger die Mob-Episode aus The Monk, um zu dem allgemeinen Schluss zu gelangen, ab 1789 reflektiere „gotischer Schrecken“ oft „revolutionären Schrecken“ (Brantlinger 1998, 50-51, hier 50 [meine Übersetzung, M. C. F.]). Spätestens an dieser Stelle sind allerdings entscheidende Präzisierungen nötig. Das post-revolutionäre Verständnis von terror bezog sich, wie gesehen, auf Staatsterrorismus nach dem Muster der Grande Terreur. Die Mob-Episode aus The Monk stellt aber gar nicht die terreur eines machthabenden Regimes dar. Statt um die inquisitionsgestützte Willkürherrschaft der Kirche, die in anderen Passagen des Romans geschildert wird, geht es hier um die Gewalthandlungen einer aufständischen Masse gegen die Kirche - also Terror ,von unten', nicht Terror ,von oben'. Brantlingers Begriff „revolutionary terror“ ist so gesehen wenig hilfreich. Ebenso problematisch ist die de Sade'sche These einer Brutalisierung und Abstumpfung der Leserschaft infolge realer Gräuel, zumal nicht alle Schauerromane von exzessiver Gewalt geprägt sind.

\section{Erhabene Unbestimmtheit: Terror versus horror}

In diesem Zusammenhang ist die Unterscheidung zwischen terror und horror von Interesse, die Radcliffe zu Beginn des 19. Jahrhunderts in einem posthum erschienenen Essay entwickelt hat. Mit terror und horror benennt Radcliffe zwei gegensätzliche ästhetische Prinzipien und zieht damit (zumindest implizit) eine Grenze zwischen zwei unterschiedlichen Schreibweisen innerhalb der Schauerliteratur, nämlich ihrer eigenen einerseits und derjenigen Lewis' andererseits. Auf den ersten Blick scheint die Gegenüberstellung von terror und horror nur schwer nachvollziehbar, da beide Begriffe oft synonym verwendet werden und sich ihre Bedeutungen überschneiden. Das Oxford English Dictionary definiert horror als „[a] painful emotion compounded of loathing and fear; a shuddering with terror and repugnance; strong aversion mingled with dread; the feeling excited by something shocking or frightful. Also in weaker sense, intense dislike or repugnance. (The prevalent use at all times.)“(OED, „Horror“) Genau wie terror kann horror mit Schrecken übersetzt werden. Im Falle von horror mischt sich dem Schrecken jedoch Aversion und Verachtung bei: Das, was uns in Angst versetzt, erscheint uns zugleich als widerwärtig, weshalb man zur besseren Differenzierung gegenüber dem Schrecken von Grausen sprechen könnte. Radcliffe geht es bei ihrer Begriffsunterscheidung jedoch weniger um die psychischen Schattierun- 
gen beider Emotionen. Vielmehr ist sie an ihrem Verhältnis zur Vorstellungskraft interessiert. Radcliffes idiosynkratischer Deutung von terror und horror zufolge kann nur ersterer Affekt die Imagination der Leserinnen und Leser anregen und dabei zur Quelle erhabener Empfindungen werden.

Der betreffende Text Radcliffes erschien 1826 im New Monthly Magazine als Fragment aus dem Nachlass der drei Jahre zuvor verstorbenen Autorin. Konzipiert war er ursprünglich als Teil der Einleitung zu Radcliffes letztem Roman Gaston de Blondeville. Im New Monthly Magazine wurde er jedoch als alleinstehender Essay veröffentlicht, mit dem (nicht von Radcliffe selbst stammenden) Titel On the Supernatural in Poetry, unter dem er bis heute bekannt ist. Das Fragment besteht weitgehend aus einem Dialog und enthält nur rudimentäre erzählerische Elemente. Auf der Reise durch Warwickshire unterhalten sich die Gesprächspartner „Mr. S-“ und „W-“ über das Übernatürliche im dramatischen Werk Shakespeares. Als sie auf das Motiv der Geistererscheinung zu sprechen kommen, lobt W- die Eröffnungsszenen aus Hamlet als Musterbeispiel für die erhabene Art und Weise, wie Shakespeare das Übernatürliche einsetze. Das bloße Reden von diesen Szenen versetze ihn in einen Zustand des erhabenen Schauers: „I thrill with delightful awe, even while I recollect and mention them, as instances of the exquisite art of the poet.“ (Radcliffe 1826, 148) Seinen Enthusiasmus für die Geistererscheinungen in Hamlet teilt W- mit dem bereits zitierten Encyclopédie-Artikel zur terreur (vgl. Jaucourt 1765, 186) sowie mit der entsprechenden Passage bei Chamfort, in der es heißt: „[C]ette scène de Hamlet [...] où le phantome paraît: il est vrai que cette scène est le chef-d'œuvre du théâtre moderne dans le genre terrible.“ [„Diese Szene im Hamlet, in welcher der Geist erscheint: Es ist wahr, dass sie das Meisterstück des modernen Theaters in der Gattung des Schrecklichen ist."] (Chamfort 1824, 191) Bei Radcliffe wird allerdings ein anderes Verständnis von terror vorausgesetzt als in den dramentheoretischen Texten der französischen Aufklärung. Für W- erzeugt die Erscheinung des Geistes von König Hamlet nicht aristotelischen phobos, sondern eine „düstere und erhabene Art des Schreckens“ (Radcliffe 1826, 149 [meine Übersetzung, M. C. F.]). Dies erläutert er mit Verweis auf Burkes Enquiry: „The union of grandeur and obscurity, which Mr. Burke describes as a sort of tranquillity tinged with terror, and which causes the sublime, is to be found only in Hamlet [sic!]; or in scenes where circumstances of the same kind prevail.“ (ebd., 149)

Gemeint sind die Passagen in Burkes Enquiry, in denen ,Dunkelheit' als zweitwichtigstes Merkmal erhabener Objekte aufgeführt wird - gleich nach dem ,Schrecken` und noch vor der ,Macht‘ (vgl. Burke 2004 [1757/1759], 102-107). Burke gebraucht den Begriff obscurity nicht nur im wörtlichen Sinne, zur Bezeichnung des Fehlens von Licht, sondern auch ich im Sinne von Unklarheit beziehungsweise Undeutlichkeit. In seinen Augen ist Dunkelheit eng mit literarischer 
Repräsentation verbunden. Im Gegensatz zur visuellen Darstellung der Malerei könne Dichtung die Unbestimmtheit der Sprache nutzen. Sie habe die Freiheit, entscheidende Details wegzulassen, abstrakte Konzepte wie Unendlichkeit und Ewigkeit $\mathrm{zu}$ verwenden und eine Vielzahl miteinander konfligierender Bilder $\mathrm{zu}$ schaffen. Ersteres und letzteres illustriert Burke am Beispiel von Miltons Versepos Paradise Lost (1667/1674), aus dem er das Porträt des Todes (2.666-2.673) sowie die erste Beschreibung Satans (1.589-1.599) zitiert. Dagegen habe die Malerei nur beschränkte Möglichkeiten, Unbestimmtheit zu erzeugen, da sie letztlich einer naturgetreuen, gegenständlichen Darstellung verpflichtet bleibe, die die repräsentierten Objekte für den Betrachter erkennbar gestalten müsse (dass Burke mit dieser Einschätzung falsch lag, braucht heute nicht mehr erwähnt zu werden; schon die Werke William Turners aus der ersten Hälfte des 19. Jahrhunderts sollten dies eindrücklich belegen). Burke war der Überzeugung, dass Klarheit in der Darstellung der Erregung von Leidenschaften im Wege steht und dass uns die Kunst der Malerei deshalb zwar Bewunderung abverlangt, nicht aber in einen Zustand erhabener Empfindungen zu versetzen vermag. Die Burkesche „Bevorzugung der Suggestion gegenüber der genauen Bestimmung“ (Trott 1998, 80 [meine Übersetzung, M. C. F.]) sollte ein wesentliches Merkmal der Ästhetik des Erhabenen bleiben, gerade auch in der Schauerliteratur. Noch Mary Shelleys 1818 erschienener Roman Frankenstein; or, The Modern Prometheus zelebriert narrative Unbestimmtheit in Bezug auf Victor Frankensteins Kreatur, deren Entstehung ebenso im Vagen bleibt wie ihre äußere Erscheinung. Der erste Ich-Erzähler Robert Walton charakterisiert das Monster einmal als ,a form which I cannot find words to describe: gigantic in stature, yet uncouth in its proportions“ (Shelley 1998 [1818], 186-187) und rekurriert dabei auf den Unaussprechlichkeitstopos, der fester Bestandteil der Ästhetik des Erhabenen ist.

Radcliffe wertet das Stilmittel der Dunkelheit in ihrem Dialog sogar noch stärker auf als Burke. Wenn das Schreckenerregende in allen Details beschrieben werde, argumentiert W- (als Sprachrohr der Autorin), bleibe für die Leserinnen und Leser nichts mehr übrig, was sie sich selbst ausmalen könnten. Vorzuziehen sei darum eine Erzählweise, die nur unvollständig darstelle, flüchtige Eindrücke beschreibe und schattenhafte Umrisse entwerfe und die dergestalt die Einbildungskraft dazu anrege, den fehlenden Rest zu ergänzen (vgl. Radcliffe 1826, 150). Ohne das Element der Dunkelheit verkomme der Schrecken (terror) zu bloßem Grausen und Entsetzen (horror), der die Geisteskräfte nicht beflügele, sondern sie im Gegenteil lähme: „Terror and horror are so far opposite, that the first expands the soul, and awakens the faculties to a high degree of life; the other contracts, freezes, and nearly annihilates them. I apprehend, that neither Shakspeare [sic!] nor Milton by their fictions, nor Mr. Burke by his reasoning, anywhere looked to positive horror as a source of the sublime, though they all agree that 
terror is a very high one; and where lies the great difference between horror and terror, but in the uncertainty and obscurity, that accompany the first [sic!], respecting the dreaded evil?““ (ebd., 149-150)

Ein Text kann demnach entweder die Möglichkeit eines schreckenerregenden Ereignisses evozieren, oder er kann die Figuren (und somit die Leserinnen und Leser) mit tatsächlichen schrecklichen Gegebenheiten konfrontieren. Im ersteren Fall beschreibt er bestimmte Indizien, die zwar als Hinweise auf ein mögliches grauenvolles Geschehen interpretiert werden können, die ein solches Geschehen aber eben nicht in aller Eindeutigkeit bestätigen. Nach Radcliffes Verständnis passt der Begriff terror nur auf erstere Textart, wohingegen letztere als Horrorerzählung beschrieben werden muss. Während Schreckensnarrative die Leserinnen und Leser dazu anregen, die Hinweise des Textes aktiv zu deuten, versetzen Horrornarrative uns in eine rein passive Haltung: Wir werden so stark von Schrecken, Ekel und Abscheu erfasst, dass unsere Vorstellungskraft vorübergehend ihre Arbeit einstellt.

Vielleicht hatte Radcliffe Passagen wie die oben diskutierte Mob-Episode aus The Monk im Sinn, wo der Lynchmord an der Priorin sehr plastisch geschildert wird: „She [i. e. the Prioress] sank upon the ground bathed in blood, and in a few minutes terminated her miserable existence. Yet though She [sic] no longer felt their insults, the Rioters still exercised their impotent rage upon her lifeless body. They beat it, trod upon it, and ill-used it, till it became no more than a mass of flesh, unsightly, shapeless, and disgusting.“ (Lewis 1998 [1796], 356) Der horror, den die Beschreibung des Lynchmordes bei den Leserinnen und Lesern auslösen soll, wird von den beobachtenden Figuren im Text selbst empfunden: „Unable to prevent this shocking event, Lorenzo and his Friends [sic!] had beheld it with the utmost horror.“ (ebd., 356) Horror durchfährt auch Manfred in The Castle of Otranto beim Anblick des riesigen Helms, der seinen fünfzehnjährigen Sohn Conrad in Stücke reißt. Um die Leserinnen und Leser an diesem Grausen teilhaben zu lassen, spricht Walpole in drastischen Worten von den „blutenden, verstümmelten Überresten des jungen Prinzen“ (Walpole 1998 [1764/1765], 19 [meine Übersetzung, M. C. F.]).

Die explizite Thematisierung von Affekten ist charakteristisch für den Schauerroman, der Schauern nicht nur erzeugen will, sondern auch zum Gegenstand hat. Viele Dutzende Male tauchen die Wörter terror und horror jeweils in den Romanen Radcliffes auf, deren Heldinnen sich durch eine ausgeprägte Empfindsamkeit auszeichnen. Dies macht sie besonders empfänglich für all das Schreckliche und Grauenerregende, dem sie im Verlauf der Handlung ausgesetzt werden, und das sie in großer Regelmäßigkeit in Ohnmacht fallen lässt. Radcliffes bekanntester Roman The Mysteries of Udolpho behält den von Walpole eingeführten ,gotischen`Schauplatz bei, stellt der unheimlichen Burg von Udolpho in den 
Apenninen, wo die Leidenszeit für die Heldin Emily St. Aubert beginnt, jedoch die zu Beginn beschriebene pastorale Idylle des bescheidenen elterlichen chateau in der Gascogne gegenüber. Der Roman ist im Jahr 1584 angesiedelt. Kurz nach Einsetzen der Handlung verliert Emily zuerst ihre Mutter und später auch ihren Vater, mit dem sie eine große Liebe zur Natur verband. Als Waise muss Emily ihrer wenig wohlgesonnenen Tante Madame Cheron in das isolierte Schloss des Heiratsschwindlers Montoni folgen, der sich als Edelmann ausgibt, in Wahrheit aber eine Bande plündernder Söldner anführt. Aus den heimlichen Aktivitäten der Banditen ergibt sich für Emily immer wieder der Eindruck unerklärlicher, scheinbar übernatürlicher Geschehnisse. Und dieser Eindruck wird durch die Geistergeschichten der abergläubischen Dienerinnen verstärkt - so sehr Emily auch versucht, ihm mit der Kraft der Vernunft entgegenzuwirken. Am berühmtesten ist das Beispiel des schwarzen Schleiers, der in einer Kammer der Burg ein ominöses Bild verhüllt. Nachdem sie den Schleier eines Nachts bemerkt hat, wird Emily von Neugierde erfasst. Geheimnisvolle Geschichten über die ehemalige Burgherrin Signora Laurentini und die Tatsache, dass niemand anders sich traut, den Schleier zu lüften, erfüllen sie mit einem Anflug von Schrecken (vgl. Radcliffe 1980 [1794], 248). Dazu wird eine Erläuterung angefügt, die - wie viele andere Passagen des Romans - geradezu lehrbuchartig die Burkesche Theorie des erhabenen Schreckens zur Anwendung bringt: „But a terror of this nature, as it occupies and expands the mind, and elevates it to high expectation, is purely sublime, and leads us, by a kind of fascination, to seek even the object, from which we appear to shrink.“ (ebd., 248) So betritt Emily schließlich alleine die Kammer und greift nach dem Schleier. In nur einem Satz wird uns mitgeteilt, dass das, was sie sieht, kein Bild ist, und dass sie noch auf dem Weg zur Tür das Bewusstsein verliert (vgl. ebd., 248). Wir befinden uns im sechsten Kapitel des Romans. Erst im siebzehnten Kapitel erfahren wir, was sich hinter dem schwarzen Schleier verbarg: etwas, das wie ein verwester und von Würmern besiedelter Leichnam aussah (vgl. ebd., 662). Dabei handelte es sich jedoch, wie sogleich nachgeschoben wird, um eine bloße Wachsfigur, die einst für einen Marquis von Udolpho hergestellt worden war - als memento mori und Strafe für mangelnden Respekt vor der Autorität der katholischen Kirche. Während Emily selbst an der betreffenden Stelle im Roman von horror übermannt wird (ein Wort, das in diesem Zusammenhang gleich zweimal fällt), bleibt für die Leserinnen und Leser vierhundert Seiten lang der terror des Geheimnisses bestehen. Wir haben ausführlich Zeit, uns vorzustellen, was Emily gesehen hat. Hätte Radcliffe schon im sechsten Kapitel die Eindrücke ihrer Heldin vom ekelerregenden Wachsleichnam beschrieben, hätte sie (ihrer eigenen Definition gemäß) einen Horror-Effekt erzielt. So aber hält die Erzählung mittels ,Dunkelheit' den erhabenen Schrecken aufrecht und regt die Vorstellungskraft der Leserinnen und Leser bis zum Ende an. 


\section{Schluss}

In ihrem Buch The Coherence of Gothic Conventions beschreibt Eve Sedgwick den Schauerroman als eine formelhafte Gattung, die auf eine geringe Zahl von Motiven beschränkt sei: „Once you know that a novel is of the Gothic kind, you can predict its contents with an unnerving certainty.“(Sedgwick 1986, 9) Schon die wenigen in diesem Beitrag betrachteten Beispiele verdeutlichen allerdings, wie heterogen der Schauerroman bereits im Zeitraum zwischen 1764 und 1818 war, ungeachtet seiner Vorliebe für südeuropäische Handlungsorte und ,gotische‘ Bauwerke und ungeachtet seiner geradezu obsessiven Auseinandersetzung mit einem Imaginären des Katholizismus' (Elementen, die allerdings in Frankenstein gänzlich fehlen). Was Manfreds Heimsuchung durch einen riesenhaften Geist mit Ambrosios Sündenfall und Teufelspakt verbindet, ist der dahinterstehende Versuch, mit literarischen Mitteln Schrecken zu erzeugen. Die unerklärlichen Ereignisse, die sich während Emilys Gefangenschaft in der Burg von Udolpho zutragen, und die Kreatur Victor Frankensteins zeigen, wie produktiv der Schreckaffekt sich auf die Einbildungskraft auswirkt und wie unterschiedlich die ,Schreckgespenster sind, die er hervorbringt. Diese „spectres, apparitions, ghosts“ (Anonymus 1798 [1797], 223) mögen zwar vereinzelt mit der politischen Gewalt und dem Terror der Französischen Revolution zusammenhängen; die von den frühen Schauerromanen etablierten Schreibweisen haben ihren ursprünglichen historischen Kontext jedoch überlebt. Und so begegnen uns terror und horror noch heute allenthalben in ihrer ,gotischen', das heißt auf erhabenen Schauer ausgerichteten Form.

\section{Literaturverzeichnis}

Anonymus. „The Terrorist System of Novel-Writing“ [1797]. Gothic Readings. The First Wave, 1764-1840. Hrsg. von Rictor Norton. London und New York, NY: Leicester University Press, 2000. 299-303. (Zuerst erschienen als „Letter to the Editor“. Monthly Magazine 4.21 (August 1797): 102-104.)

Anonymus. „Terrorist Novel Writing“. The Spirit of the Public Journals for 1797. Being an Impartial Selection of the Most Exquisite Essays and Jeux d'Esprits, Principally Prose, That Appear in the Newspapers and Other Publications. With Explanatory Notes and Anecdotes of Many of the Persons Alluded to. Bd. 1. London: R. Philipps, 1798. 223-225.

Aristoteles. Poetik. Griechisch/Deutsch. Übers. und hrsg. von Manfred Fuhrmann. Stuttgart: Reclam, 1997.

Boileau-Despréaux, Nicolas. Euvres diverses du sieur $D^{\star \star *}$. Avec le Traité du sublime ou du merveilleux dans le discours, traduit du Grec de Longin. Paris: Claude Barbin, 1764.

Brantlinger, Patrick. The Reading Lesson. The Threat of Mass Literacy in Nineteenth-Century British Fiction. Bloomington, IN: Indiana University Press, 1998. 
Burke, Edmund. „A Philosophical Enquiry into the Origin of Our Ideas of the Sublime and the Beautiful“ [1757/1759]. A Philosophical Enquiry into the Origin of Our Ideas of the Sublime and the Beautiful and Other Pre-Revolutionary Writings. Hrsg. von David Womersley. London: Penguin, 2004. (Deutsche Fassung: Philosophische Untersuchung über den Ursprung unserer Ideen vom Erhabenen und Schönen. Übers. von Friedrich Bassenge. Hrsg. von Werner Strube. 2. Aufl. Hamburg: Meiner, 1989).

Byron, Glennis, und Dale Townshend (Hrsg.). The Gothic World. London und New York, NY: Routledge, 2014.

Chamfort, Sébastien Roch Nicolas. Ébauches d'une poétique dramatique. Euuvres de Chamfort. Bd. 4. Hrsg. von P. R. Auguis. Paris: Chez Chaumerot Jeune, Libraire, 1824. 1-234.

Clery, Emma J. „The Supernatural Explained“. The Rise of Supernatural Fiction, 1762-1800. Cambridge: Cambridge University Press, 1995. 106-114.

Clery, Emma J. „The Genesis of ,Gothic“ Fiction“. The Cambridge Companion to Gothic Fiction. Hrsg. von Jerrold E. Hogle. Cambridge: Cambridge University Press, 2002. 21-39.

D’Arnaud, François-Thomas-Marie de Baculard. „Préface [à Fayel]“. đEuvres de d’Arnaud. Contenant Fayel. Bd. 10. Paris: Laporte, 1795. iii-xl.

Freud, Sigmund. „Das Unheimliche“ [1919]. Gesammelte Werke. Hrsg. von Anna Freud, Marie Bonaparte, E. Bibring und W. Hoffer. Bd. 12. Frankfurt am Main: Fischer, 1999. 227-268.

Fuhrmann, Manfred. „Nachwort“. Aristoteles. Poetik. Griechisch/Deutsch. Übers. und hrsg. von Manfred Fuhrmann. Stuttgart: Reclam, 1997. 144-178.

Grimm, Jacob, und Wilhelm Grimm. Deutsches Wörterbuch von Jacob Grimm und Wilhelm Grimm. Bd. 14: $R$ - Schiefe. Hrsg. von Dr. Moriz Heyne. München: Deutscher Taschenbuch Verlag, 1999 [Reprint von Deutsches Wörterbuch von Jacob Grimm und Wilhelm Grimm. Bd. 8: $R$ - Schiefe. Hrsg. von Dr. Moriz Heyne. Leipzig: S. Hirzel, 1893].

Heuvel, Gerd van den. „Terreur, Terroriste, Terrorisme“. Handbuch politisch-sozialer Grundbegriffe in Frankreich 1680-1820, Heft 3: Philosophe, Philosophie/Terreur, Terroriste, Terrorisme. Hrsg. von Rolf Reichardt und Eberhard Schmitt. München: R. Oldenbourg, 1985. 89-132.

Hurd, Richard. Letters on Chivalry and Romance. London: Printed for A. Millar, in the Strand; and W. Thurlbourn and J. Woodyer, in Cambridge, 1762.

Jackson, Rosemary. Fantasy. The Literature of Subversion. London und New York, NY: Routledge, 1981.

Jaucourt, Le Chevalier [Louis] de. „Terreur“. Encyclopédie ou Dictionnaire raisonné des sciences, des arts et des métiers. Par une société de gens de lettres. Mis en ordre et publié par $M$ r. ***. Tome Sezième [sic!]: Te - Venerie. Neufchatel: Samuel Faulche \& Compagnie, 1765. 186.

Kelly, George Armstrong. „Conceptual Sources of the Terror“. Eighteenth-Century Studies 14.1 (1980): 18-36.

Keßler, Helmut. Terreur. Ideologie und Nomenklatur der revolutionären Gewaltanwendung in Frankreich von 1770 bis 1794. München: Fink, 1973.

Lewis, Matthew. The Monk. Hrsg. von Howard Anderson. Oxford und New York, NY: Oxford University Press, 1998 [1796].

Longinus. Vom Erhabenen. Griechisch/Deutsch. Übers. und hrsg. von Otto Schönberger. Stuttgart: Reclam, 1997.

Monk, Samuel. The Sublime. A Study of Critical Theories in XVIII-Century England. Ann Arbor: The University of Michigan Press, 1960 [1935].

OED. „Gothic“. The Oxford English Dictionary. URL: http://www.oed.com/view/Entry/80225. 
OED. „Horror“. The Oxford English Dictionary. URL: http://www.oed.com/view/Entry/88577.

OED. „Terror“. The Oxford English Dictionary. URL: http://www.oed.com/view/Entry/199606.

OED. „Terrorist“. The Oxford English Dictionary. URL: http://www.oed.com/view/Entry/199609.

Paulson, Ronald. „Gothic Fiction and the French Revolution“. English Literary History 48.3

(1981): 532-554.

Radcliffe, Mrs. [Ann]. „On the Supernatural in Poetry“. The New Monthly Magazine and Literary Journal, Part I: Original Papers 16.1 (1826): 145-152.

Radcliffe, Ann. The Mysteries of Udolpho. Hrsg. von Bonamy Dobrée. Oxford und New York, NY: Oxford University Press, 1980 [1794].

Sade, D. A. F. de. Idée sur les romans [1800]. Euvres complètes. Bd. 4. Les crimes de l'amour. Bd. 1. Paris: Jean-Jacques Pauvert, 1961. 11-43.

Scott, Sir Walter. Rezension zu ,Fatal Revenge; or, the Family of Montorio: a Romance'. The Quarterly Review, May 1810: 339-347.

Sedgwick, Eve Kosofsky. „The Structure of Gothic Conventions“. The Coherence of Gothic Conventions. New York, NY und London: Methuen, 1986. 9-36.

Shelley, Mary. Frankenstein or The Modern Prometheus. The 1818 Text. Hrsg. von Marilyn Butler. Oxford und New York, NY: Oxford University Press, 1998.

Tatar, Maria M. „The Houses of Fiction: Toward a Definition of the Uncanny“. Comparative Literature 33.2 (1981): 167-182.

Todorov, Tzvetan. Introduction à la littérature fantastique. Paris: Seuil, 1970.

Trott, Nicola. „The Picturesque, the Beautiful and the Sublime“. A Companion to Romanticism. Hrsg. von Duncan Wu. Oxford und Malden, MA: Blackwell, 1998. 72-90.

Walpole, Horace. The Castle of Otranto. Hrsg. von Wilmarth Sheldon Lewis. Oxford und New York, NY: Oxford University Press, 1998 [1764/1765].

Walther, Rudolf. „Terror, Terrorismus“. Geschichtliche Grundbegriffe. Historisches Lexikon zur politisch-sozialen Sprache in Deutschland. Bd. 6. Hrsg. von Otto Brunner, Werner Conze und Reinhart Koselleck. Stuttgart: Klett-Cotta, 1990. 351-444. 